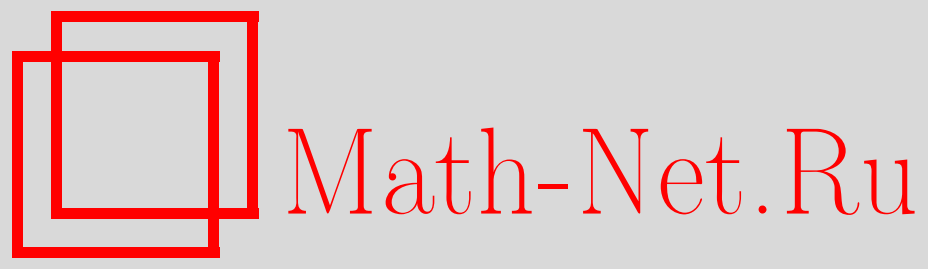

Л. Д. Фаддеев, Пентагон Волкова для модулярного квантового дилогарифма, Функи. анализ и его прил., 2011, том 45, выпуск 4, 65-71

DOI: https://doi.org/10.4213/faa3054

Использование Общероссийского математического портала MathNet.Ru подразумевает, что вы прочитали и согласны с пользовательским соглашением

http://www . mathnet.ru/rus/agreement

Параметры загрузки:

IP: 18.234 .197 .8

26 апреля 2023 г., 13:37:45

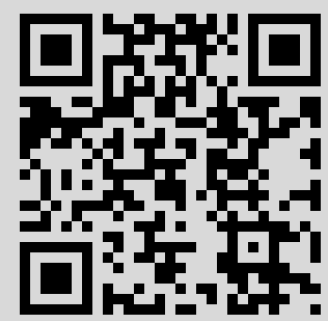




\title{
Пентагон Волкова для модулярного квантового дилогарифма*
}

\author{
(C) 2011. Л. Д. ФАДДЕЕВ \\ Посвящаю памяти моего многолетнего друга Димы Арнольда
}

Новая форма уравнений пентагона, предложенная Волковым [1] для $q$-экспоненты на основе формальных рядов, выводится в рамках гильбертова пространства для модулярного варианта квантового дилогарифма.

Функция

$$
\begin{aligned}
e(x) & =1+\sum_{n=1}^{\infty} \frac{q^{n(n-1) / 2}}{\left(q^{-1}-q\right) \cdots\left(q^{-n}-q^{n}\right)} x^{n} \\
& =\prod_{n=0}^{\infty}\left(1+q^{2 n+1} x\right)
\end{aligned}
$$

известна еще со времен Эйлера. Менее популярно ее представление в виде

$$
e(x)=\exp \sum_{n=1}^{\infty} \frac{x^{n}(-1)^{n}}{n\left(q^{n}-q^{-n}\right)} .
$$

Здесь в знаменателе суммы стоит целое число $n$ и его $q$-аналог, так что естественно говорить о q-дилогарифме или, следуя современной моде, о квантовом дилогарифме. Это становится особенно естественным, если использовать $e(x)$ в некоммутативном контексте.

Пусть $U, V$ образуют вейлеву пару:

$$
U V=q^{2} V U
$$

Шютценберже показал [2], что

$$
e(U) e(V)=e(U+V) .
$$

В работе [3] Волков и я вывели формулу

$$
e(V) e(U)=e\left(U+V+q^{-1} U V\right)
$$

откуда, в силу (4), следует пентагональное соотношение

$$
e(V) e(U)=e(U) e\left(q^{-1} U V\right) e(V) .
$$

* Работа написана при поддержке программы РАН «Математические проблемы нелинейной динамики», гранта 09-01-93108-НЦНИЛа и гранта РФФИ 11-01-00570.

3 Функциональный анализ и его приложения, т. 45, вып. 4 
В [4] Кашаев и я показали, что эта формула является некоммутативным аналогом пятичленного соотношения для дилогарифма Роджерса $\mathrm{L}(x)$

$$
\mathrm{L}(x)+\mathrm{L}(y)=\mathrm{L}\left(\frac{x(1-y)}{1-x y}\right)+\mathrm{L}(x y)+\mathrm{L}\left(\frac{y(1-x)}{1-x y}\right),
$$

где $x, y$ - переменные из интервала $[0,1]$. Доказательство основано на квазиклассическом вычислении в пределе $q \rightarrow 1$ с использованием асимптотики

$$
e(u) \sim \exp \left\{\frac{1}{2 \ln q} E(u)\right\}, \quad E(u)=\operatorname{Li}_{2}(-u),
$$

где $\mathrm{Li}_{2}(u)$ - дилогарифм Эйлера. Функции $\mathrm{L}(x)$ и $\mathrm{Li}_{2}(x)$ связаны соотношением

$$
\mathrm{L}(x)=\mathrm{Li}_{2}(x)+\frac{1}{2} \ln x \ln (1-x) .
$$

Последний результат вызывает некоторые вопросы:

1. Почему в некоммутативной формуле участвует дилогарифм Эйлера, а в классическом пределе стоит дилогарифм Роджерса?

2. Почему аргументы функций в формулах (5) и (6) так различаются?

Ответ на первый вопрос обсуждается уже в [4], и окончательный ответ найден в работе [5], где подробно исследуется квазиклассическая асимптотика в формуле (5) и ее обобщениях, возникающих в конформной теории поля. Очень красивый ответ на второй вопрос дан в работе Волкова [1], где пентагональное соотношение было переписано в новой форме.

Начнем с классического случая. Пусть

$$
R(u)=\mathrm{L}\left(\frac{u}{1+u}\right) .
$$

Легко проверить, что соотношение (6) эквивалентно равенству

$$
R(u)+R(v)=R\left(\frac{v}{1+u}\right)+R\left(\frac{u v}{1+u+v}\right)+R\left(\frac{u}{1+v}\right) .
$$

Аргументы, участвующие здесь,

$$
x_{1}=u, \quad x_{2}=v, \quad x_{3}=\frac{1+v}{u}, \quad x_{4}=\frac{1+u+v}{u v}, \quad x_{5}=\frac{1+u}{v},
$$

являются решением $Y$-системы $A_{2}$

$$
x_{i} x_{i+2}=1+x_{i+1},
$$

которая имеет период 5 ,

$$
x_{i+5}=x_{i} .
$$

Таким образом, (7) можно записать в виде

$$
R\left(x_{1}\right)+R\left(x_{2}\right)=R\left(\frac{1}{x_{5}}\right)+R\left(\frac{1}{x_{4}}\right)+R\left(\frac{1}{x_{3}}\right) .
$$

Квантовый аналог системы (8) имеет вид

$$
X_{i} X_{i+2}=1+q X_{i+1},
$$

и если

$$
X_{1}=U, \quad X_{2}=V, \quad U V=q^{2} V U,
$$


TO

$$
\begin{aligned}
& X_{3}=U^{-1}(1+q V), \\
& X_{4}=U^{-1}\left(q^{-1}+U+V\right) V^{-1}, \\
& X_{5}=(1+q U) V^{-1} .
\end{aligned}
$$

Квантовая формула Волкова имеет вид

$$
e\left(X_{1}\right) e\left(X_{2}\right)=e\left(X_{5}^{-1}\right) e\left(X_{4}^{-1}\right) e\left(X_{3}^{-1}\right) .
$$

Мы видим замечательную аналогию между формулами (9) и (12).

Рассуждения Волкова основаны на действиях с формальными рядами. Для погружения его вывода в рамки гильбертова пространства возникает препятствие. Инволюция

$$
U^{*}=U, \quad V^{*}=V
$$

требует условия $|q|=1$, в то время как исходные формулы $(1),(2)$ не определены для рационального $\ln q /(i \pi)$.

Для преодоления этой трудности я в работе [7] предложил заменить функцию $e(u)$ на ее модулярный аналог

$$
\Phi(u)=\frac{\prod\left(1+q^{2 n+1} u\right)}{\prod\left(1+\tilde{q}^{2 n+1} \tilde{u}\right)},
$$

где

$$
q=e^{i \pi \tau}, \quad \tilde{q}=e^{-i \pi / \tau}, \quad \tilde{u}=u^{1 / \tau} .
$$

В этой работе мы покажем, что формула (12) верна при замене $e(u)$ на $\Phi(u)$, и дадим ее вывод, основанный на решении $Y$-системы (10).

Пусть

$$
\gamma(z)=\exp \left\{-\frac{1}{4} \int_{-\infty}^{\infty} \frac{e^{i t z}}{\sin \omega t \sin \omega^{\prime} t} \frac{d t}{t}\right\},
$$

где контур интегрирования обходит сингулярность при $t=0$ сверху. Здесь, следуя Волкову [8], мы используем обозначения времен Вейерштрасса $\omega, \omega^{\prime}$ для периодов решетки с образующими 1 и $\tau$,

$$
\tau=\frac{\omega^{\prime}}{\omega}, \quad \omega \omega^{\prime}=-\frac{1}{4} .
$$

Интеграл в (13) имеет смысл для $\omega$ и $\omega^{\prime}$ из верхней полуплоскости, положительному $\tau$ отвечают чисто мнимые $\omega, \omega^{\prime}$. Нетрудно проверить, что

$$
\Phi\left(e^{-i \pi z / \omega}\right)=\gamma(z)
$$

Формулу (14) примем за определение модулярного квантового дилогарифма. Функция $\gamma(z)$, так же как и $e(u)$, хорошо известна в анализе. Среди многочисленных ее названий есть термин «двойная Г-функция Барнса» [9]. Ее свойства, включающие некоммутативные версии, подробно описаны в статье Волкова [8] и в ряде работ Кашаева ([10], [11]). Я ввел ее в [6] для описания дуальности в дискретной группе Гейзенберга-Вейля, а в [7] использовал для определения модулярного дубля для квантовой группы $S L_{q}(2)$, где она заменяет $q$-экспоненту в определении универсальной $R$-матрицы Дринфельда [12]. В работах [13], [14] 
она используется для описания эволюционного оператора для квантовых интегрируемых систем на дискретном пространстве-времени, а в [16] дано определение фундаментальной $R$-матрицы для XXZ модели произвольного спина. Дальнейшие ее приложения, в том числе и в математике, приведены во введении работы [5].

Перечислим свойства функции $\gamma(z)$, которые будут использоваться в дальнейшем.

1. Функциональное уравнение

$$
\frac{\gamma\left(z+\omega^{\prime}\right)}{\gamma\left(z-\omega^{\prime}\right)}=1+e^{-i \pi z / \omega} .
$$

Заметим, что справедливо аналогичное свойство при взаимной замене $\omega$ и $\omega^{\prime}$, но нам оно не будет нужно.

2. Формула обращения

$$
\gamma(z) \gamma(-z)=e^{i \beta} e^{i \pi z^{2}}, \quad \beta=\frac{\pi}{12}\left(\tau+\frac{1}{\tau}\right) .
$$

3. Унитарность

$$
\overline{\gamma(z)}=\frac{1}{\gamma(z)}
$$

при $\tau>0$ и вещественных $z$.

4. Квазиклассическая асимптотика

$$
\gamma(z) \sim \exp \left\{\frac{1}{2 \pi i \tau} E\left(e^{-i \pi z / \omega}\right)\right\} .
$$

Из формулы (15) следует соотношение

$$
\frac{\Phi(q u)}{\Phi\left(q^{-1} u\right)}=\frac{1}{1+u} .
$$

Теперь мы можем переходить к построению эволюционного оператора для квантовой $A_{2}$ системы. Реализуем операторы $U$ и $V$ на плотной области определения в $L_{2}(\mathbb{R})$ формулами

$$
\begin{aligned}
& U f(z)=e^{-i \pi z / \omega} f(z), \\
& V f(z)=f\left(z+2 \omega^{\prime}\right) .
\end{aligned}
$$

Мы встречаем здесь умножение на неограниченную функцию и сдвиг в комплексную плоскость. Область определения $\mathscr{D}$ операторов $U$ и $V$ должна разрешать такое действие. Достаточно считать, что $\mathscr{D}$ содержит целые функции от $z$, убывающие в направлениях, параллельных вещественной оси, как $e^{-z^{2}}$. При $\tau>0$ операторы $U$ и $V$ неотрицательны и существенно самосопряженные. То же справедливо для операторов $X_{3}, X_{4}, X_{5}$ из (11).

Введем оператор $\Phi$,

$$
\Phi f(z)=\Phi\left(e^{-i \pi z / \omega}\right) f(z)=\gamma(z) f(z),
$$

и преобразование Фурье

$$
F f(z)=\int_{-\infty}^{\infty} e^{-2 \pi i z t} f(t) d t
$$


Заметим, что

$$
\begin{aligned}
U F & =F V, \\
V F & =F U^{-1}, \\
V \Phi(U) & =\Phi(U)\left(1+q^{-1} U\right) V .
\end{aligned}
$$

Последнее свойство следует из функционального уравнения (18). Теперь нетрудно видеть, что

$$
X_{i+1}=S^{-1} X_{i} S
$$

где унитарный оператор $S$ дается формулой

$$
S=\Phi F
$$

Периодичность $i \equiv i+5$ будет обеспечена, если

$$
S^{5}=e^{i \alpha} I \text {. }
$$

Покажем, что это действительно так. Пусть $S^{5}(x, y)$ - ядро оператора $S^{5}$, pacсматриваемого как интегральный оператор. По определению

$$
\begin{aligned}
S^{5}(x, y)=\gamma(x) & \int \exp \left\{-2 \pi i\left(x t_{1}+t_{1} t_{2}+t_{2} t_{3}+t_{3} t_{4}+t_{4} y\right)\right\} \\
& \times \gamma\left(t_{1}\right) \gamma\left(t_{2}\right) \gamma\left(t_{3}\right) \gamma\left(t_{4}\right) d t_{1} d t_{2} d t_{3} d t_{4}=\int M(x, z) e^{2 \pi i z(x-y)} d z
\end{aligned}
$$

где

$$
\begin{aligned}
M(x, z)=\int \exp \left\{-2 \pi i\left(x t_{1}+t_{1} t_{2}+\right.\right. & \left.\left.t_{2} t_{3}+t_{3} z+x z\right)\right\} \\
& \times \gamma\left(t_{1}\right) \gamma\left(t_{2}\right) \gamma\left(t_{3}\right) d t_{1} d t_{2} d t_{3} \gamma(x) \gamma(z)
\end{aligned}
$$

Подобные интегралы в [8], [10], [15] интерпретированы как непрерывные аналоги биномиальных соотношений Рамануджана, и из приведенных там явных формул следует, что $M(x, z)$ не зависит от $x$ и $z$ и

$$
M(x, z)=e^{i \alpha}, \quad \alpha=\frac{\pi}{4}\left(\tau+\frac{1}{\tau}+1\right) .
$$

Мы видим, что

$$
S^{5}(x, y)=e^{i \alpha} \delta(x-y)
$$

Заметим теперь, что в силу формулы обращения (16) мы можем дать альтернативную форму для оператора эволюции

$$
S=e^{i \beta} \hat{\Phi}^{-1} G, \quad \beta=\frac{\pi}{12}\left(\tau+\frac{1}{\tau}\right),
$$

где

$$
\hat{\Phi} f(z)=\Phi\left(e^{i \pi z / \omega}\right) f(z)=\gamma(-z) f(z)
$$

И

$$
G=e^{i \pi z^{2}} F
$$

Легко проверить, что

$$
G^{3}=e^{i \pi / 4} F^{2}
$$


Мы имеем теперь все средства для доказательства формулы Волкова в виде

$$
\Phi\left(X_{1}\right) \Phi\left(X_{2}\right)=\Phi\left(X_{5}^{-1}\right) \Phi\left(X_{4}^{-1}\right) \Phi\left(X_{3}^{-1}\right) .
$$

Используя действие (19) оператора $S$, перепишем это соотношение в виде

$$
\Phi S^{-1} \Phi S=S^{-4} \hat{\Phi} S^{4} S^{-3} \hat{\Phi} S^{3} S^{-2} \hat{\Phi} S^{2}
$$

или вследствие определений (20) и (24)

$$
\Phi F^{-1}=S^{-4} G^{3} e^{3 i \beta}=e^{-i \alpha+3 i \beta} \Phi F G^{3},
$$

и это равенство справедливо вследствие определения $\alpha$ и $\beta$ в (23), (24), формулы (25) и тождества

$$
F^{4}=I \text {. }
$$

Итак, мы доказали основной результат (26) и убедились, что он эквивалентен формуле (21).

В заключение убедимся, что формула (23) дает классическое тождество для дилогарифма Роджерса. Мы перейдем к квазиклассическому вычислению в формуле (22). Используя асимптотику (17) и оставляя в экспоненте подынтегрального выражения только коэффициенты при $1 /(2 \pi i \tau)$, мы получаем интеграл

$$
M(x, y) \sim \int \exp \left\{\frac{1}{2 \pi i \tau} \sum_{i=1}^{5}\left(E\left(e^{p_{i}}\right)+p_{i} p_{i+1}\right)\right\} d p_{3} d p_{4} d p_{5}
$$

где

$$
p_{1}=-\frac{i \pi x}{\omega}
$$

и аналогично возникают аргументы $p_{2}, \ldots, p_{5}$ из $y, t_{1}, t_{2}, t_{3}$, a $p_{6}=p_{1}$. Используя соотношение

$$
\frac{d}{d p} E\left(e^{p}\right)=-\ln \left(1+e^{p}\right)
$$

получаем уравнения стационарной фазы

$$
\begin{aligned}
& \ln \left(1+e^{p_{3}}\right)=p_{2}+p_{4}, \\
& \ln \left(1+e^{p_{4}}\right)=p_{3}+p_{5}, \\
& \ln \left(1+e^{p_{5}}\right)=p_{4}+p_{1},
\end{aligned}
$$

которые совпадают с тремя уравнениями $Y$-системы для $x_{i}=e^{p_{i}}$, выражающие $x_{3}, x_{4}$ и $x_{5}$ через $x_{1}=x$ и $x_{2}=y$.

Теперь, переписывая сумму $\sum_{i} p_{i} p_{i+1}$ в виде $\sum_{i} \frac{1}{2} p_{i}\left(p_{i-1}+p_{i+1}\right)$, получаем квазиклассический результат

$$
\sum E\left(x_{i}\right)+\frac{1}{2} \ln x_{i} \ln \left(1+x_{i}\right)=-\frac{\pi^{2}}{2}=-3 \frac{\pi^{2}}{6},
$$

что приводит к формуле (9), если учесть, что

$$
R(x)=-E(x)-\frac{1}{2} \ln x \ln (1+x) \quad \text { и } \quad \frac{\pi^{2}}{6}-R(x)=R\left(\frac{1}{x}\right) .
$$

Мы явно видим, как происходит замена эйлерова дилогарифма на дилогарифм Роджерса. 
Большинство результатов в этой работе в той или иной мере возникли при обсуждении с А. Ю. Волковым и Р. М. Кашаевым. В литературе, посвященной использованию квантового дилогарифма, можно найти некоторые из приведенных здесь результатов, см., например, [17], [18]. Здесь я просто собрал их в одну из возможных последовательных логических схем. Я благодарен Волкову и Кашаеву за их участие в обсуждениях. Работа дозрела и была написана в прекрасных условиях, созданных мне в Греции профессорами Котсиолисом, Саввиди и Зупанасом. Всем им я приношу глубокую благодарность.

\section{ЛитеРАТУРА}

[1] A. Yu. Volkov, Pentagon identity revisited, Int. Math. Res. Notices (2011); doi:10. 1093/imrn/rnr200; http://arxiv.org/abs/1104.2267.

[2] M. P. Schützenberger, Une interprétation de certaines solutions de l'équation fonctionnelle $F(x+y)=F(x) F(y)$, C. R. Acad. Sci. Paris, 236 (1953), 352-353.

[3] L. D. Faddeev, A. Y. Volkov, Abelian current algebra and the Virasoro algebra on the lattice, Phys. Lett. B, 315 (1993), 311-318; http://arxiv.org/abs/hep-th/9307048.

[4] L. D. Faddeev, R. M. Kashaev, Quantum dilogarithm, Modern. Phys. Lett. A, 9:5 (1994), 427-434; http://arxiv.org/abs/hep-th/9310070.

[5] R. Kashaev, T. Nakanishi, Classical and quantum dilogarithm identities, http:// arxiv.org/abs/1104.4630.

[6] L. D. Faddeev, Discrete Heisenberg-Weyl group and modular group, Lett. Math. Phys., 34:3 (1995), 249-254.

[7] L. D. Faddeev, Modular double of quantum group, Math. Phys. Stud., 21 (2000), 149156; http://arxiv.org/abs/math/9912078.

[8] A. Yu. Volkov, Noncommutative hypergeometry, http://arxiv.org/abs/math/0312084.

[9] E. W. Barnes, The genesis of the double gamma function, Proc. London Math. Soc., 31 (1899), 358-381.

[10] R. M. Kashaev, Liouville central charge in quantum Teichmuller theory, http:// arxiv.org/abs/hep-th/9811203.

[11] R. M. Kashaev, Quantization of Teichmueller spaces and the quantum dilogarithm, Lett. Math. Phys., 43:2 (1998), 105-115.

[12] В. Г. Дринфельд, Квантовые группы, Зап. научн. семин. ЛОМИ, 155 (1986), 1949.

[13] L. Faddeev, A. Yu. Volkov, Hirota equation as an example of integrable symplectic map, Lett. Math. Phys., 32:2 (1994), 125-135; http://arxiv.org/abs/hep-th/9405087.

[14] L. D. Faddeev, R. M. Kashaev, A. Y. Volkov, Strongly coupled quantum discrete Liouville theory. 1. Algebraic approach and duality, Comm. Math. Phys., 219:1 (2001), 199-219; http://arxiv.org/abs/hep-th/0006156.

[15] B. Ponsot, J. Teschner, Clebsch-Gordan and Racah-Wigner coefficients for a continuous series of representations of $U_{q}(s l(2, R))$, Comm. Math. Phys., 224:3 (2001), 613-655; http://arxiv.org/abs/math.QA/0007097.

[16] А. Ю. Волков, Л. Д. Фаддеев, Квантовый метод обратной задачи на дискретном пространстве-времени, ТМФ, 92:2 (1992), 207-214; preprint HU-TFT-93-30.

[17] L. Chekhov, V. Fock, Quantum Teichmüller spaces, http://arxiv.org/abs/math.QA/ 9908165.

[18] A. B. Goncharov, The pentagon relation for the quantum dilogarithm and quantized $M_{0,5}$, http://arxiv.org/abs/0706.4054v2.

Санкт-Петербургское отделение

Математического Института

им. В. А. Стеклова РАН

e-mail: faddeev@pdmi.ras.ru 